\title{
Study of Role of Magnetic Resonance Urography (MRU) and Comparison with Conventional Radiology in the Diagnosis of Complex Renal Anomalies: A Tertiary Care Centre Experience
}

\author{
Priti Zade, Sharad Khandelwal, Milind Joshi, Sandesh Parelkar, Shyam Borwankar \\ Opposite Bombay Hospital, Indore, India \\ Email: milindj79@yahoo.com
}

Received May 4, 2012; revised June 20, 2012; accepted July 4, 2012

\begin{abstract}
Anatomical renal anomalies are one of the most commonly occurring renal anomalies. Most of these anomalies require series of investigations for the proper diagnosis. We tried to compare the results of conventional radiological investigations with magnetic resonance urography in the diagnosis of complex renal anomalies. Materials and methods: This was a prospective study done over a period of five years from 2006 to 2011. All the patients with suspected or diagnosed presence of renal anomalies were investigated by ultrasound (USG), Intravenous urography (IVU), micturating cystourethrogram (MCU), magnetic resonance urography (MRU), retrograde urethrography, cystogenitoscopy, renal scans as per the indication in the case. Results: Total sixty three patients were studied over the period. The most common complex renal anomaly diagnosed was duplex system and conventional radiological investigations were useful in the diagnosis of less than $50 \%$ patients. Female patients outnumbered male patients in having complex renal anomalies. MRU was diagnostic in most of the patients with such anomalies and excellent mode of investigation for functional and anatomical details. Conclusion: MRU is better than conventional radiological investigations in the diagnosis of complex renal anomalies.
\end{abstract}

Keywords: Complex Renal Anomalies; Conventional Radiology; Magnetic Resonance Urography

\section{Introduction}

Anatomical renal anomalies are one of the most commonly occurring renal anomalies [1]. However, they are not perfectly diagnosed all the times either because some are asymptomatic or not detected by any single modality of conventional radiological investigation. Such patients require battery of conventional investigations and still may not be diagnosed even after that. Hence there is a need for a single, noninvasive and authoritative imaging which will help in finding out these anomalies. With this purpose, we carried out study comparing role of magnetic resonance urography (MRU) with conventional radiological investigations over a period of five years at a tertiary care centre in patients suspected with or diagnosed with complex anatomical renal anomalies in age group up to 12 years of age.

\subsection{Aims and Objectives}

1) To study patients of urological diseases with complex renal anomalies using magnetic resonance urogra- phy (MRU) as a diagnostic tool;

2) To compare the findings of MRU with findings of conventional radiological investigations;

3) To confirm the findings of investigative modalities with intra operative findings;

4) To study the utility of MRU in the diagnosis of congenital or complex urological structural anomalies;

5) Analysis of observations of the study.

\subsection{Materials and Methods}

This was a prospective study done over a period of five years from 2006 to 2011.

All the patients of renal pathologies were initially screened with ultrasound (USG) as a baseline investigation. Those patients who were diagnosed with congenital renal anomalies or were suspected to have renal anomalies but were not diagnosed on USG, underwent intravenous urography (IVU), micturating cystourethrography (MCU), retrograde urography and Cystogenitoscopy as per indications using standard protocols.

All the patients underwent MRU after explaining the 
procedure and obtaining proper consent for the procedure. The study of MRU was carried out on 1.5 Tesla magnetic field closed MRI.

Very young and uncooperative patients were given oral sedation in the form of trichlorfon (pedicloryl) in the dose of $50 \mathrm{mg} / \mathrm{kg}$. Intravenous fluids were given as per body weight to maintain proper hydration. Intravenous gadolinium contrast was used for the study for image acquisition using abdominal or body coil, with patient in supine position and coil positioned over upper abdomen and centered on kidneys. After initial localizing images were obtained in the following sequences

T2 HASTE single slice

T2 HASTE multislice

3D GRE T1

TRUFI 2D

FL2D 80

TSE FS T2

Post contrast images in T1 sequences were obtained.

Intravenous urography (IVU) was performed in suspected congenital structural or complex renal anomalies using $1,3,5,7,15,30,45$ min sequences as per requirement. Delayed 24-hour plates were taken in nonfunctioning or poorly functioning kidney.

Nuclear scintigraphy renal scans with diuretics were done in the cases of obstructive uropathy.

\subsection{Evaluation of MR Images}

MR images were examined to determine the presence or absence of dilatation in the urinary system, to find out functional status of renal unit, to find out cause of dilatation and its site, to see anatomical details of kidneys and duplex units if present, anatomy of the ureter and ectopic ureter if there were more than one collecting system, to define the contours of urinary bladder and other associated congenital renal anomalies. These findings were compared with findings of conventional radiological investigations and confirmed during surgical procedure if surgical intervention was done.

\section{Results}

Total sixty three patients were studied. The distribution of patients and findings in each patients are summarized in the Table 1. Complex renal anomalies were defined as those having one or more anomalies, bilateral anomalies, when the anomalies required more than one conventional radiological investigation for the diagnosis or were not diagnosed even after the use of these investigations. Conventional radiological investigations were useful in the diagnosis of less than $50 \%$ patients in cases of duplex renal anomalies which was by far the commonest congenital anomaly in our study. Three patients had bilateral duplex renal system.
Ten patients underwent upper pole heminephrectomy for nonfunctioning upper moiety. One patient had both the moieties functioning and hence underwent upper pole ureteric reimplantation for the refluxing moiety. Two patients had pathological single unit on one side and normally functioning duplex unit on the other side and underwent nephrectomy of the pathological single unit of the opposite side.

Two patients are under regular follow up and are asymptomatic and on chemoprophylaxis and doing well and two patients did not turn up for further follow up. One patient had unilateral duplex with ureterocele of the upper moiety which was normally functioning and incision of ureterocele was done. In cases of single system ureteral dysplasia, again MRU was diagnostic in all the patients and diagnosis was not possible in any patient by IVU and was suspected only in 50\% patients after USG. All these patients underwent laparoscopic nephroureterectomy for the dysplastic kidneys because of the symptom of incontinence in spite of normal voiding pattern. The similar pattern was noticed in other renal anomalies with the use of conventional investigations and MRU. The patients of bilateral ectopic ureter and ureteric valves underwent ureteric reimplantation after the successful diagnosis. The patients of ureterocele underwent endoscopic incision of the ureterocele because of good renal functional parenchyma and are under regular follow up. The patients of MCDK are also under follow up. The patients with pelviureteric junction obstruction underwent pyeloplasty.

Incidentally, two or more conventional investigations were suggesting similar diagnosis in cases where they were diagnostic for the presence of anomalies. The details of the various anomalies found in the study were as mentioned in the observation Table 1.

The incidence of anomalies was more in the female group. The acquired complex conditions like renal abscesses or pyelonephritis were also commoner in females.

\section{Discussion}

The purpose of the study was to compare the diagnostic utility of MRU in comparison to conventional investigations and was fulfilled well. The more number of female patients having congenital and acquired renal problems was consistent with findings of other studies in the literature $[2,3]$.

Duplication is the most common congenital anomaly in the urinary tract, a $0.7 \%$ incidence in one series [4]. During initial screening, the diagnosis of duplex system may be possible by ultrasound evaluation. It can also detect associated renal dysplasia, hydronephrotic changes of upper or lower moiety and associated ureterocele if present [5]. However, it is highly observer dependent and 
Table 1. Distribution and type of anomalies detected in the study.

\begin{tabular}{|c|c|c|c|c|c|}
\hline$N=63$ & MRU & IVU & USG & $\mathrm{MCU}$ & Symptomatic $(\mathrm{Y} / \mathrm{N})$ \\
\hline Duplex renal system (22) & 22 (Female-14) & 10 & 6 & - & $\mathrm{Y}$ \\
\hline Single system ureteral ectopia (9) & 8 (Male-1) & - & 4 & - & $\mathrm{Y}$ \\
\hline Bilateral ectopic ureter (2) & 2 (Female) & 2 & 2 & 2 & $\mathrm{Y}$ \\
\hline Primary megaureter (3) & 2 (Female) & 3 & 2 & 2 & $\mathrm{Y}$ \\
\hline Pelvi ureteric junction obstruction (3) & 3 (Male-1) & - & 1 & - & $\mathrm{Y}$ \\
\hline Uretero-vesical junction obstruction (3) & 3 (Male-1) & 1 & 2 & - & Y \\
\hline Ureteric valve (1) & 1 (Male) & - & - & - & $\mathrm{Y}$ \\
\hline Multicystic dysplastic kidney (2) & 2 (Male-1) & - & 1 & - & $\mathrm{N}$ \\
\hline Cystic renal disease (1) & 1 (Female) & - & - & - & $\mathrm{Y}$ \\
\hline Ureterocele (2) & 2 (Male-1) & 1 & - & 1 & $\mathrm{Y}$ \\
\hline Ectopic kidney (3) & 3 (Female) & - & 1 & - & $\mathrm{Y}$ \\
\hline Pyelonephritis (4) & 4 (Female-3) & - & 1 & - & $\mathrm{Y}$ \\
\hline PU valve with complications (2) & 2 & - & 2 & 2 & $\mathrm{Y}$ \\
\hline Renal abscess/UTI (6) & 6 (Female-4) & 2 & 2 & - & $\mathrm{Y}$ \\
\hline
\end{tabular}

many times duplex anomaly can be missed and also fails to identify duplex moieties when associated with hydroureter.

MCU can diagnose associated refluxing unit in cases of duplex moiety and Ureterocele. However as a single investigation, MCU cannot diagnose presence of duplex system.

IVU which is considered as a standard investigation for morphological assessment of renal unit fails to identify nonfunctioning upper moieties of duplex system.

Abnormal alignment of upper pole calices of lower moiety because of pressure effect of dysplastic upper moiety can be seen on IVU which may give clue to the diagnosis.

Renal scintigraphy is necessary to know functional status of the duplex moieties which influence the treatment. It however gives poor anatomical delineation. In 1996, Pearlman et al. described the concept of CT Urography (CTU) as a diagnostic method in renal diseases. Multislice CT with contrast and 3D reconstruction also gives excellent anatomical details but has high radiation exposure and risk of allergy to the contrast [6].

We found that, MRU as a single investigation gives excellent anatomical description of the duplex renal moieties even when they are nonfunctioning. After applying the simple chi square statistical analysis for the assessment ability of MRU as a single modality of investigations in comparison to the conventional radiological investigations for the diagnosis of complex renal anomalies, the probability of chance occurrence was less than $0.5 \%$. MRU is a non invasive examination method that does not entail ionizing radiation and does not require iodinated contrast as in IVU or CTU and safer in children. It is excellent in identifying nonfunctioning or poorly functioning duplex system.

The heavily weighted T2 images best pick up the dilated non functioning moieties using static fluid as hyperintence images (Figure 1). This technique does not require contrast [7]. The T1-weighted images pick up non dilated and functioning unit as hyperintense (Figure 2). Development of faster sequences, the GRE-T1 weighted contrast sequences provide the opportunity for faster examination. It is possible to obtain IVU-like images by employing Maximum Intensity Projection (MIP) Method on heavily T2 weighted images and contrast enhanced T1 images [8-12].

We attribute the reasons for failure of IVU to diagnose An ectopic ureter with congenital renal dysplasia classically presents in female patient as continence with incontinence [1]. It is one of the surgically curable causes of incontinence, hence require proper diagnosis and surgery.

IVU is not useful in the diagnosis of single system ureteral ectopia with renal dysplasia as there is no excretion of the contrast on the affected side. USG as an initial screening method along with color Doppler is used to rule out duplex system and to find out presence of dysplastic kidney and its localization either at ectopic site or in the renal fossa and to visualize the renal vascularity. It can also detect associated hydroureter if system is obstructed. However it is highly observer dependent and the dysplastic renal tissue may not be easy to see as was clearly evident in our cases where in only two out of seven cases, dysplastic kidney was detected, giving it a sensitivity of $30 \%$ only.

Renal scans are not very useful in diagnosing such cases as the dysplastic renal tissue has hardly any function and such systems are usually obstructed rather than 


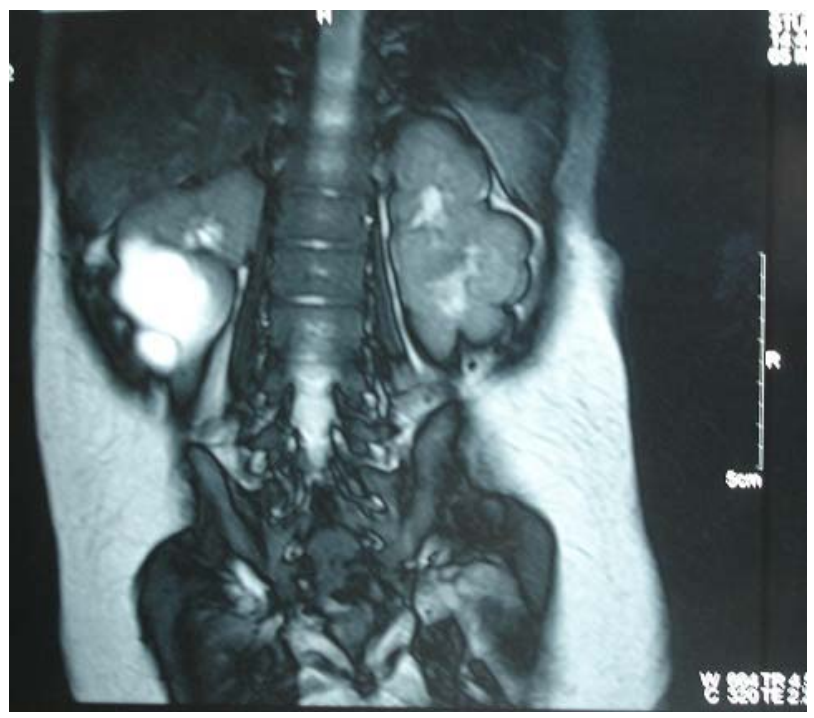

Figure 1. T2W image of the right duplex hydro nephrotic system showing hyper intensity suggestive of dilated and non functioning moiety.

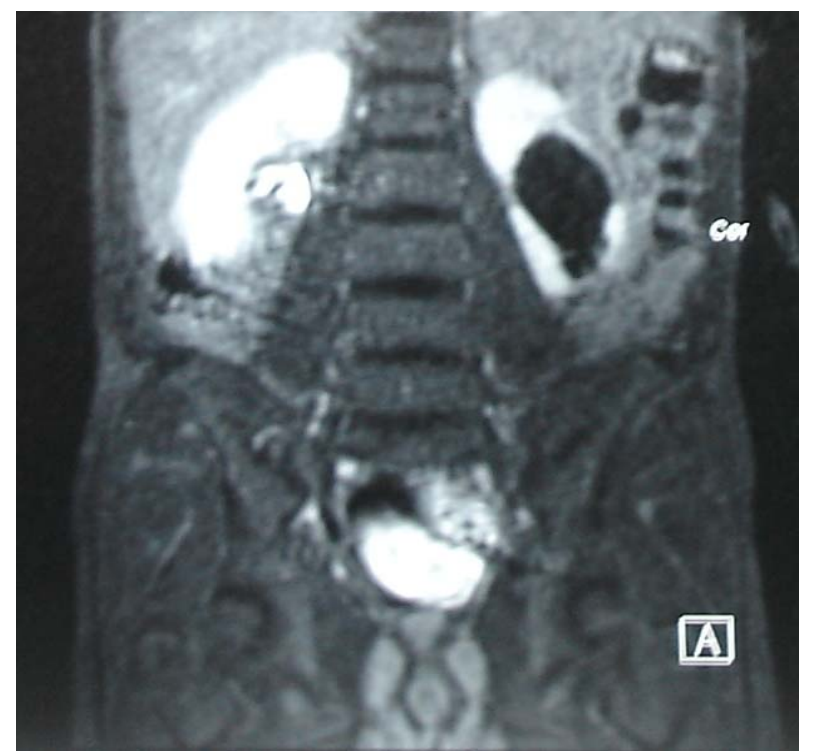

Figure 2. T1W image showing hyper intensity is suggestive of non dilated functioning system.

the duplex system where operative intervention was not required to presence of ectopic moiety superimposed on the bony structures and could not be seen in IVU, borderline functioning, poor patient preparation and where the pressure from the dilated lower moiety was obstructing the drainage as well as compromising the perfusion of the duplex moiety and hence was appearing like non functioning moiety on IVU. Sometimes, the anatomical arrangement of the calyces is so close to each other that on IVU they resembled as calyces of the single unit and misinterpreted by the radiologist and only MRU could detect the duplex in this. refluxing system. In our cases also these investigations did not help which was contrary to the findings of the series reported by Gangopadyaya et al. where DMSA renal scans and IVU were diagnostic modalities in their series of patients [6].

In females, this single system ectopic ureter opens in the vestibule, vagina, cervix or distal urethra and thus produces symptom of incontinence with otherwise normal pattern of voiding. Because of the stenotic opening of this ureter and its insertion outside the urinary bladder, this ureter mostly is not associated with reflux and VCUG is almost always normal in this population and subsequently is not useful for the diagnosis of the single system ectopic ureter with renal dysplasia. In this population, it is the functional status of the associated renal unit which is important from the management point of view rather than the presence or absence of reflux in the ureter.

CT urography with contrast with $3 \mathrm{D}$ reconstruction of the images has also been used as an investigation in some of the published studies [2,7]. However, it has the dis advantage of very high radiation exposure and risk of allergic reaction to the contrast agent and hence we did not use this investigation in our cases.

MRU is an excellent modality of investigation in such cases [8]. The dysplastic renal tissue is specifically picked up on T1W and T2W images and course of the ectopic ureter could also be seen very well in all of the seven cases. The T2W images picked up the dysplastic kidney and the hydroureter as hyper intense images as in $\mathrm{T} 2 \mathrm{w}$ images the dilated system appears as hyperintence and in T1W images nonfunctioning system appear hypo intense [9-14]. Gadolinium contrast images gave excellent picture of functioning renal tissue and ureter and could be differentiated from non functioning tissue [15-18]. 3D reconstruction with maximum intensity projection (MIP) of the images gave very clear anatomical and functional picture of normal as well as pathological system like none of the conventional imaging (Figure 3) [19-21]. It also had the advantage of no radiation exposure and as a single modality of investigation could detect the ectopic ureter with dysplastic kidney [22,23].

In cases of pelviureteric junction obstruction, in addition to giving anatomical details MRU helps in diagnosing the abnormal vasculature also [24-27]. It can also be used to quantify the renal function and can avoid the need for renal scinti scans $[27,28]$.

In patients of multi cystic dysplasia of kidney (MCDK), the MRU gives excellent anatomical description which is not possible in any of the conventional investigations.

The average cost of MRU in our country is approximately 100 USD and average time period for one patient is 30 minutes.

With the availability and increasing use of MRU this cost aspect will definitely get reduced. Even then, the 


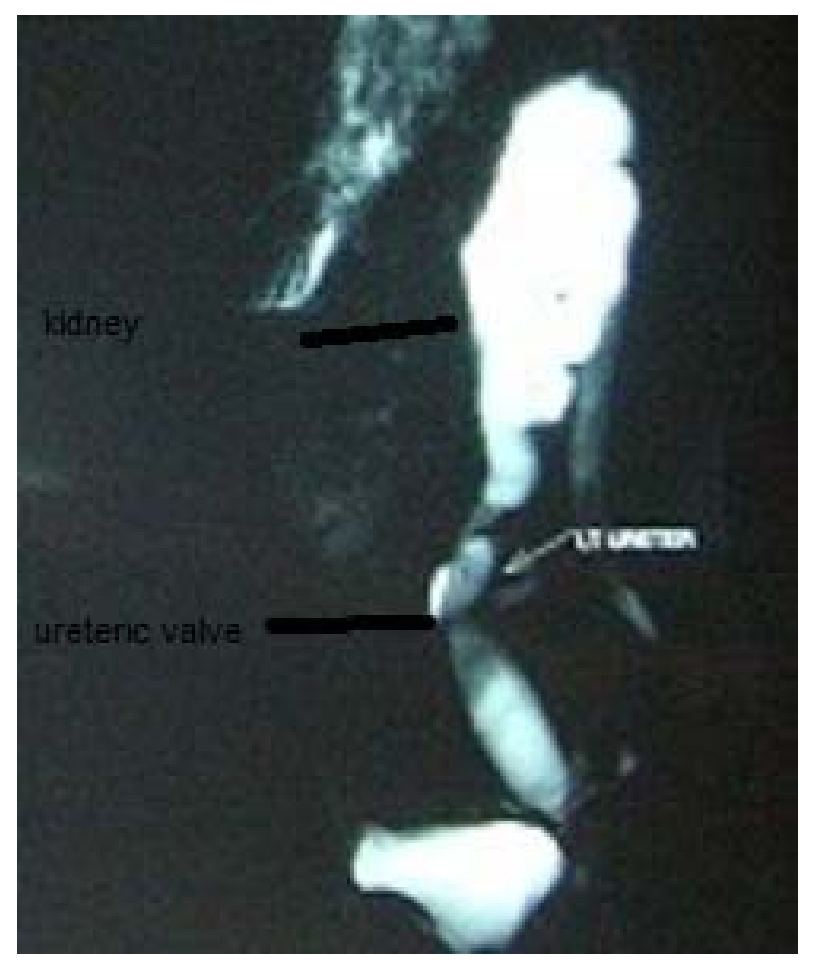

Figure 3. Maximum intensity projection (MIP) 3D reconstruction images MRU showing excellent anatomical details in an obstructed left renal system due to distal ureteral valve.

information gained by the MRU is precious in complex renal anomalies and MRU cannot be denied only for the cost factor. Increasing technological advances will also reduce the time period for the single scan.

\section{Conclusion}

In our study of complex renal anomalies we found MRU to be definitely better investigation for diagnosis than the IVU and nuclear scintigraphy. It can reduce the time, cost and help in treating the complex renal anomalies in a much better way in pediatric population.

\section{REFERENCES}

[1] C. C. A. Nolte-Ernsting, et al., "MR Uroghraphy Today," Abdominal Imaging, Vol. 28, No. 2, 2003, pp. 191-209. doi:10.1007/s00261-001-0187-4

[2] G. A. Verswijvel, et al., "Magnetic Resonance Imaging in the Assessment of Urological Disease: An All in One Approach," European Radiology, Vol. 10, No. 10, 2000, pp. 1614-1619. doi:10.1007/s003300000536

[3] C. C. A. Nolte-Ernsting, et al., "MR Urography: Examination Technique and Clinical Applications," European Radiology, Vol. 11, No. 3, 2001, pp. 355-372. doi: $10.1007 / \mathrm{s} 003300000685$

[4] G. Sigmund, et al., "RARE MR Urography in the Diagnosis of Upper Urinary Tract Abnormalities in Children,"
Pediatric Radiology, Vol. 21, No. 6, 1991, pp. 416-420. doi:10.1007/BF02026676

[5] P. Aerts, et al., "Breath Holding MR Urography Using the Haste Technique," American Journal of Roentgenology, Vol. 166, 1996, pp. 543-546.

[6] F. Regan, et al., "MR Urography Using HASTE Imaging in the Assessment of Ureteric Obstruction," American Journal of Roentgenology, Vol. 167, 1996, pp. 11151120 .

[7] A. Rothpearl, et al., "MR Urography: Technique and Application," Radiology, Vol. 194, No. 1, 1995, pp. 125130.

[8] M. E. O'Malley, et al., "MR Urography: Evaluation of the Three Dimensional Fast Spin Echo Technique in Patients with Hydronephrosis," American Journal of Roentgenology, Vol. 168, No. 2, 1997, pp. 387-392.

[9] M. Tsubota, et al., "Utility of Cine MR Urography of Urinary Tract and Comparison with Static MR Urography," Radiation Medicine, Vol. 22, 2004, pp. 212-217.

[10] K. Szopinsky, et al., "Magnetic Resonance Urography: Initial Experience Low Dose GD DTPA Enhanced Technique," European Radiology, Vol. 10, 2000, pp. 10581064.

[11] J. Hughes, et al., "MR Urography: Evaluation of Different Techniques in Non Dilated Tracts," Clinical Radiology, Vol. 57, No. 11, 2002, pp. 989-994. doi: $10.1053 /$ crad.2002.1070

[12] T. El Diasty, et al., "Diuretic Contrast Enhanced Magnetic Resonance Urography versus Intravenous Urography for Dipiction of Non Dilated Urinary Tracts," $A b$ dominal Imaging, Vol. 28, No. 1, 2003, pp. 135-145. doi:10.1007/s00261-002-0010-x

[13] F. B. Ergen, et al., "3D Excretory MR Urography: Improved Image Quality with Intravenous Saline and Diuretic Administration," Journal of Magnetic Resonance Imaging, Vol. 25, No. 4, 2007, pp. 783-789. doi: $10.1002 /$ jmri.20875

[14] C. C. A. Nolte-Ernsting, et al., "Gadolinium Enhanced Excretory Urography after Low Dose Diuretic Injection: Comparison with Conventional Excretory Urography," Radiology, Vol. 209, 1998, pp. 147-157.

[15] A. Karabacakoglu, et al., "Diagnostic Value of Diuretic Enhanced Excretory MR Urography in Patients with Obstructive Uropathy," European Journal of Radiology, Vol. 52, No. 3, 2004, pp. 320-327. doi:10.1016/j.ejrad.2003.10.023

[16] J. Zielonko, et al., "MR Urography of Obstructive Uropathy: Diagnostic Value of the Method in Selected Clinical Groups," European Journal of Radiology, Vol. 13, No. 4, 2003, pp. 802-809.

[17] F. E. Avni, et al., "The Role of MR Imaging for the Assessment of Complicated Duplex Kidneys in Children: Preliminary Report," Pediatric Radiology, Vol. 31, No. 4, 2001, pp. 215-223. doi:10.1007/s002470100439

[18] A. Borthne, et al., "MR Urography: The Future Gold Standard in Pediatric Urogenital Imaging?" Pediatric Radiology, Vol. 29, No. 9, 1999, pp. 694-701. doi: $10.1007 / \mathrm{s} 002470050677$ 
[19] J. D. Gratten-Smith, et al., "MR Urography in Children," Pediatric Radiology, Vol. 36, No. 11, 2006, pp. 11191132. doi:10.1007/s00247-006-0222-2

[20] M. Riccabona, "Pediatric MRU: Its Potential and Its Role in the Diagnostic Work-Up of the Upper Urinary Tract Dilatation in Infants and Children," World Journal of Urology, Vol. 22, No. 2, 2004, pp. 79-87. doi:10.1007/s00345-004-0406-Z

[21] M. Riccabona, et al., "Feasibility of MR Urography in Neonates and Infants with Abnormalities of the Upper Urinary Tract," European Radiology, Vol. 12, No. 6, 2002, pp. 1442-1450. doi:10.1007/s00330-001-1180-6

[22] R. A. Jones, et al., "Dynamic Contrast Enhanced MR Urography in the Evaluation of Pediatric Hydronephrosis. I. Functional Assessment," American Journal of Roentgenology, Vol. 185, No. 6, 2005, pp. 1598-1607. doi:10.2214/AJR.04.1540

[23] W. C. W. Chu, et al., "Dynamic Gadolinium Enhanced Magnetic Resonance Urography for Assessing Drainage in Dilated Pelvicalyceal Systems with Moderate Renal Function: Preliminary Results and Comparison with Diuresis Renography," BJU International, Vol. 93, No. 6, 2004, pp. 830-834.
doi:10.1111/j.1464-410X.2003.04725.x

[24] J. Haustein, et al., "Renal Tolerance of Gadolinium DTPA/Dimeglumine in Patients with Chronic Renal Failure," Investigative Radiology, Vol. 27, 1992, pp. 153-156. doi:10.1097/00004424-199202000-00012

[25] T. Grobner, "Gadolinium: A Specific Trigger for the Development of Nephrogenic Fibrosing Dermopathy and Nephrogenic Systemic Fibrosis?" Nephrology Dialysis Transplantation, Vol. 21, No. 4, 2006, pp. 1104-1108. doi:10.1093/ndt/gfk062

[26] D. R. Broom, et al., "Gadodiamide-Associated Nephrogenic Systemic Fibrosis: Why Radiologists Should Be Concerned," American Journal of Roentgenology, Vol. 188, No. 2, 2007, pp. 586-592.

[27] A. Blandino, et al., "MR Urography of the Ureter," American Journal of Roentgenology, Vol. 179, No. 5, 2002, pp. 1307-1314.

[28] B. B. McDaniel, et al., "Dynamic Contrast Enhanced MR Urography in the Evaluation of Pediatric Hydronephrosis. Anatomic and Functional Assessment of Ureteropelvic Junction Obstruction," American Journal of Roentgenology, Vol. 185, No. 6, 2005, pp. 1608-1614. doi:10.2214/AJR.04.1574 